\title{
MiR-26b suppresses cell proliferation and invasion by targeting cyclooxygenase 2 in human glioblastoma
}

\author{
Qiyong Jiang ${ }^{1, *}$, Yimin Liu ${ }^{2,}{ }^{,}$, Shijuan Zhang ${ }^{3}$, Naikun Li $^{2}$, Gaoling Sun ${ }^{1}$ \\ ${ }^{1}$ Department of Neurosurgery, Yidu Central Hospital, Weifang Medical University, Qingzhou 262500, Shandong, P.R. China \\ ${ }^{2}$ Department of Neurology, Yidu Central Hospital, Weifang Medical University, Qingzhou 262500, Shandong, P.R. China \\ ${ }^{3}$ Department of Intensive Care Unit, Yidu Central Hospital, Weifang Medical University, Qingzhou 262500, Shandong, \\ P.R. China \\ * These authors have contributed equally to this work as the co-first author \\ Correspondence to: Qiyong Jiang, email: jiangqiyongwfyidu@163.com
}

Keywords: miR-26b, COX-2, GBM

Received: August 29, 2016

Accepted: October 05, 2016

Published: October 17, 2016

\section{ABSTRACT}

MiRNAs are emerging as important epigenetic modulators of multiple target genes, leading to abnormal cellular signaling involving cellular proliferation in cancers. Aberrant miRNA expression has been observed in human glioblastoma (GBM). The present study was to evaluate the expression and molecular mechanisms of COX-2 and miR-26b in human GBM tissues and GBM cell lines T98G, U87 and U251. In the present study, we found that expression of miR-26b was markedly downregulated in GBM cell lines and human GBM tissues, compared to matched non-tumor associated tissues. Furthermore, miR-26b expression was inversely proportional to that of COX-2 mRNA and protein. Ectopic expression of miR-26b dramatically reduced the proliferation, colony formation, and proliferation/apoptosis-related proteins in GBM cells. Flow cytometry analysis showed that ectopic expression of miR-26b significantly decreased the percentage of $S$ phase cells and increased the percentage of G1/G0 phase cells. Finally, luciferase reporter assay revealed that miR-26b inhibited the expression of COX-2 by binding to the 3'-UTR of COX-2 in GBM cells. Taken together, our results suggest that $\mathbf{m i R}-26 \mathrm{~b}$ plays an important role to inhibit the proliferation and invasion of GBM cells, and presents a novel mechanism for direct miR-26bmediated suppression of COX-2 in GBM. Thus, miR-26b/COX-2 may have an important role in treatment of GBM patients.

\section{INTRODUCTION}

Gliomas have been reported as the most common one of primary cerebral tumors in central neural system. According to the regulation of World Health Organization (WHO) classification stantard, all gliomas can be divided into 4 grades on the basis of their histology [1-2]. Although some latest therapy technologies (resection and radio-/chemotherapy) have been introduced into the treatment of gliomas, the whole effects on patients with gliomas remain to be not satisfactory [3-4]. The glioblastoma multiforme (GBM) acts as grade IV glioma, and has the most common and malignant properties [56]. Due to the unsatisfactory survival status of patients with GBM, thus it is essential to hunt for some new and useful biomarker, which can be applied into diagnosis and treatment.

MicroRNAs act as a kind of small and non-coding RNAs with the length of about 20 nucleotides, and take part in the regulation of gene expression. Mechanically, miRNAs can bind the 3'-unstranslated regions (3'-UTR) of the related target mRNA sequences, and then suppresses gene transcription or facilitate the degradation of mRNA $[7,8]$. To date, miRNAs have been demonstrated to play important roles in a wide range of oncogenic activities, including proliferation, apoptosis, angiogenesis, and metastasis [9-12]. Although the miRNA-induced potential regulation mechanisms are still under investigation, some current reports have indicated that the expression model of miRNA will be useful for the diagnosis and prognosis of 
human tumors. Notably, it has been reported that miR-26b has a wide role in regulation of some functions involving suppression of cancer cell proliferation and inhibition of organ metastasis of different cancers. At the same time, the expression of miR-26b was found to be downregulated in some cancers, inducing chronic myelogenous leukemia, gastric cancer, and colorectal carcinoma [1315]. However, little is known about the role of miR-26b in the development of GBM.

In the present study, we demonstrated that the expression of miR-26b was extremely down-regulated in GBM cells and samples; however its expression was not changed in their own adjacent non-tumor samples. Using in-vitro assay, we found that miR-26b regulates GBM cell proliferation by directly targeting 3'-UTRs of COX-2, and the over-expression of miR-26b altered the proliferative and invasive property. Our findings indicated that the inhibited miR-26b expression can enhance the proliferation and invasion of GBM cells.

\section{RESULTS}

\section{The expression of COX-2 in GBM tissues and lines}

To well understand the mechanisms underlying miR-26b-induced tumor biology and miR-26b-COX-2 pathway, we firstly detected the expression model and status of COX-2 in human GBM tissues using the realtime RT-PCR and western blot. Our findings showed that the expression of COX-2 mRNA was significantly up-regulated in cancer tissues, however, the expression was not changed in their own adjacent non-tumor tissues $(\mathrm{p}<0.001)$ (Figure 1a). In addition, results from western blot analysis revealed that the expression of COX-2 protein was also significantly up-regulated in cancer tissues, however, the expression was not changed in their own adjacent non-tumor tissues $(\mathrm{p}<0.001)$ (Figure $1 \mathrm{~b})$. Next we further detected the expression model and status of COX-2 in GBM cell lines, including T98G, U87 and U251. All cells were subjected to the real-time RT-PCR and western blot. Our findings showed that the expression of COX-2 mRNA and protein were indeed obviously higher as compared with their own adjacent non-tumor tissues (all $\mathrm{p}<0.001$ ) (Figure 2a, 2b).

\section{The expression of miR-26b in GBM tissues and cell lines}

To well understand the mechanisms underlying miR-26b-induced tumor biology and miR-26b-COX-2 pathway, we secondly detected the expression model and status of miR-26b in human GBM tissues using the real-time RT-PCR. Real-time RT-PCR analyses revealed that the expression of miR-26b was significantly downregulated in representative GBM tissues, and GBM cell lines including T98G, U87 and U251. However, the expression was not changed in their own adjacent nontumor tissues (all $\mathrm{p}<0.001$ ) (Figure 3a). To identify whether the downregulation of miR-26b can be found in all GBM
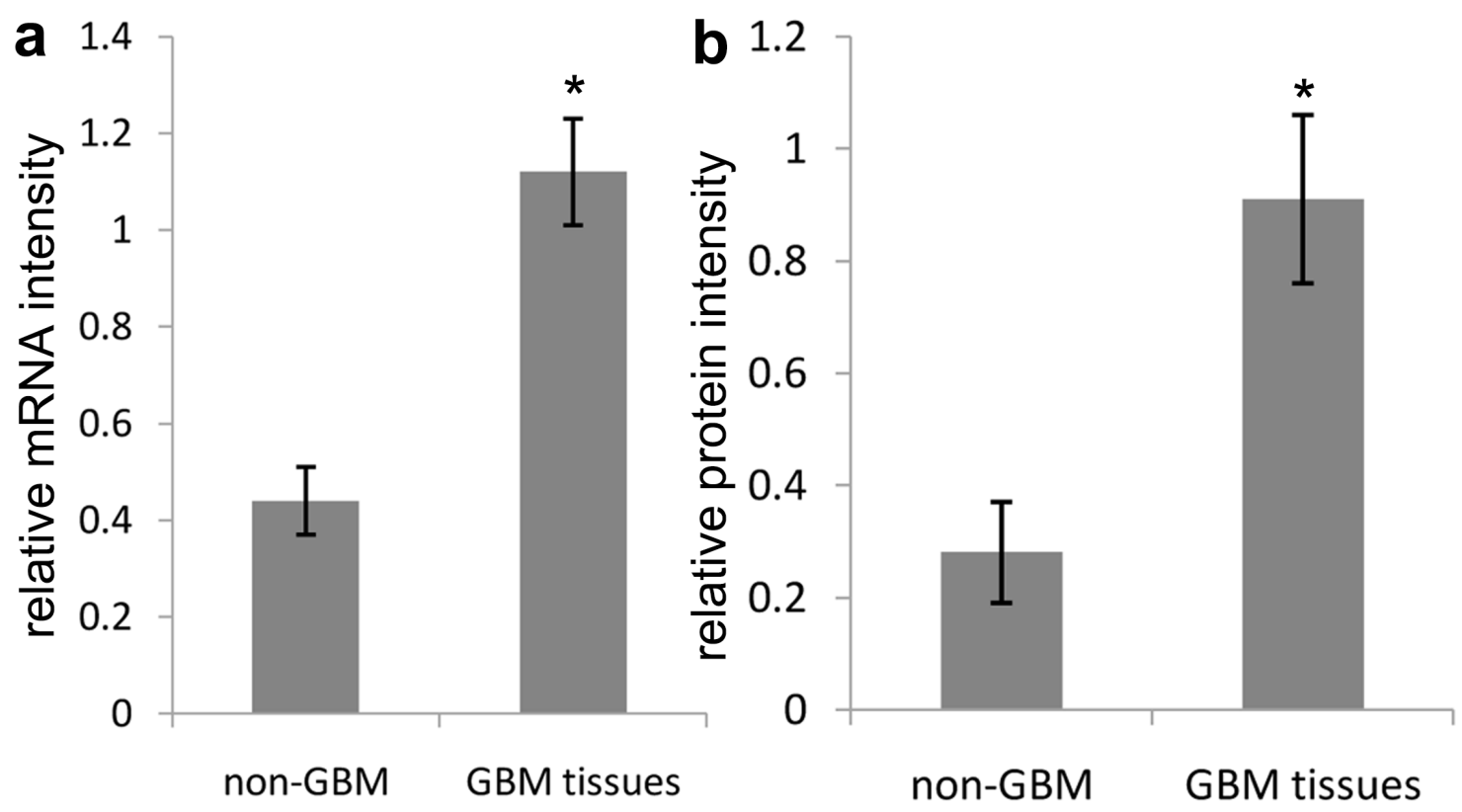

Figure 1: Analysis of COX-2 expression in GBM tissues. a. Real-time RT-PCR analysis of COX-2 mRNA expression in 30 pairs of human GBM tissues. Quantification analysis was defined as the relative density of COX-2 mRNA to GAPDH. GAPDH was used as an internal control. Results shown are the mean \pm SEM of repeated independent experiments. ${ }^{*} p<0.001$, compared with control, one-way ANOVA. b. The expression of COX-2 protein was examined in 30 pairs of human GBM tissues using western blot. The average COX2 protein expression was normalized to $\beta$-actin. Results shown are the mean \pm SEM of repeated independent experiments. ${ }^{*} p<0.001$, compared with control, one-way ANOVA. 


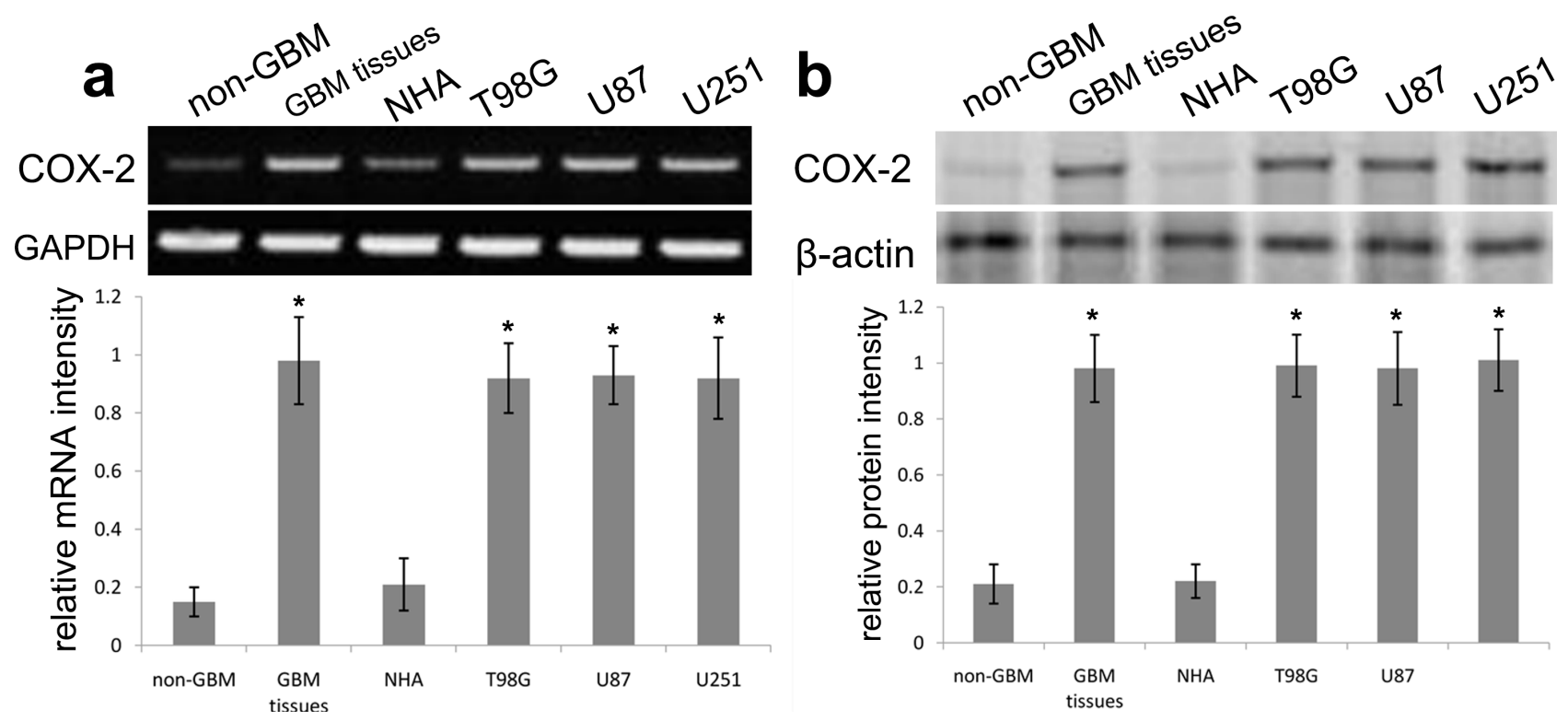

Figure 2: Analysis of COX-2 expression in GBM cell lines. a. Real-time RT-PCR analysis of COX-2 mRNA expression in representative GBM tissues and cell lines, including T98G, U87 and U251. Quantification analysis was defined as the relative density of COX-2 mRNA to GAPDH at each time point. GAPDH was used as an internal control. Results shown are the mean \pm SEM of repeated independent experiments. ${ }^{*} \mathrm{p}<0.001$, compared with control, one-way ANOVA. b. The expression of COX-2 protein was examined in representative GBM tissues and cell lines, including T98G, U87 and U251 using western blot. The average COX-2 expression was normalized to $\beta$-actin expression. Results shown are the mean \pm SEM of repeated independent experiments. ${ }^{*} \mathrm{p}<0.001$, compared with control, one-way ANOVA.
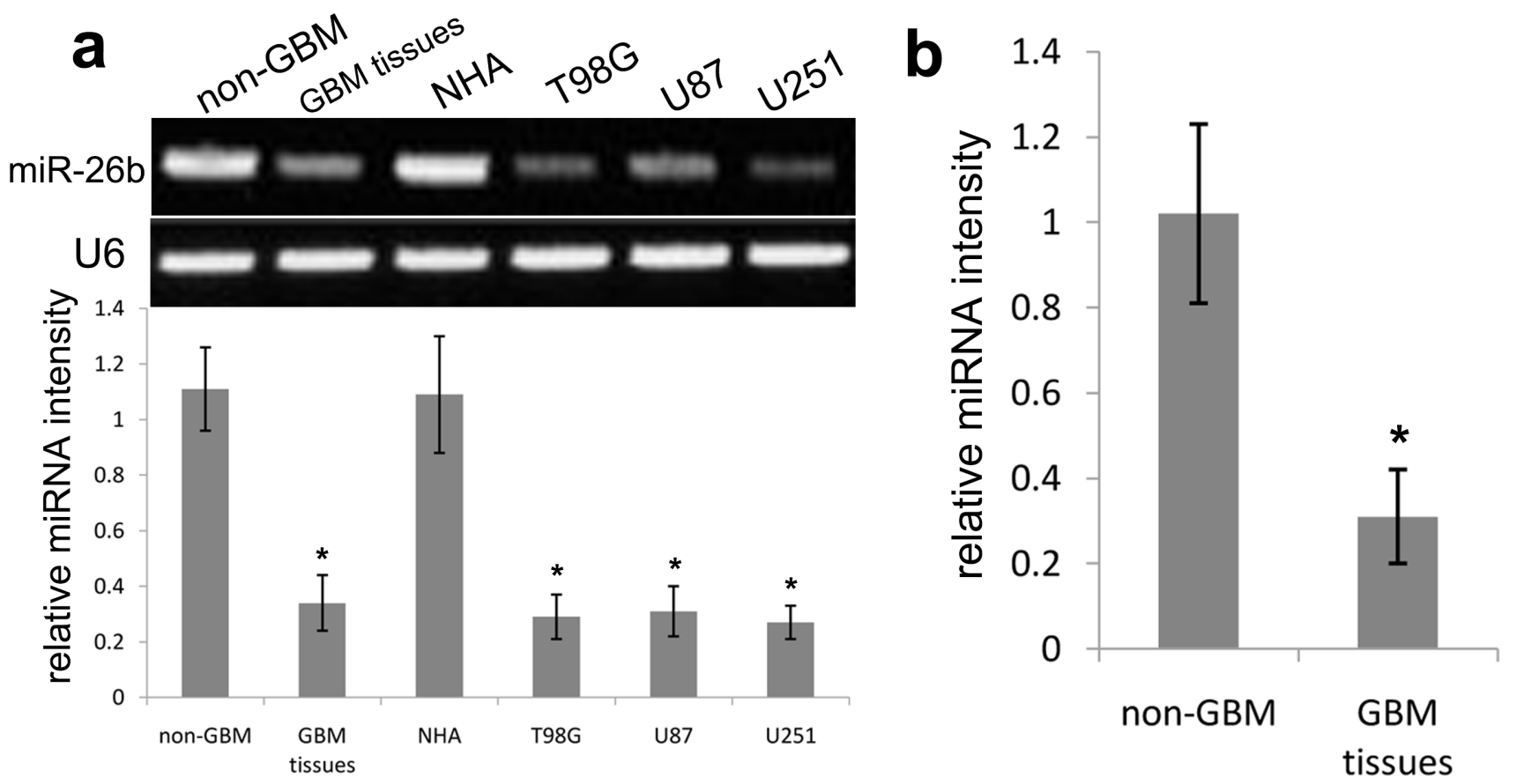

Figure 3: Reduced miR-26b expression in GBM cell lines and tissues. a. Relative miR-26b expression in representative GBM tissues and GBM cell lines (T98G, U87 and U251) was detected using real-time RT-PCR. The average miR-26b expression was normalized to U6 expression. Each bar represents the mean of three independent experiments. ${ }^{*} \mathrm{p}<0.001$, compared with control, one-way ANOVA. b. The expression of miR-26b was examined in all 30 pairs of GBM tissues using real-time RT-PCR. The average miR-26b expression was normalized to U6 expression. Each bar represents the mean of three independent experiments. * $<<0.001$, compared with control, one-way ANOVA. 
tissues, we also detected the expression of miR-26b in all 30 GBM tissues and their own adjacent non-tumor tissues. As shown in Figure 3b, our findings showed that the expression of miR-26b was significantly reduced in all GBM tumor tissues. However, the expression was not changed in their own adjacent non-tumor tissues (all p<0.001). Taken together, our findings indicated that the expression of miR$26 \mathrm{~b}$ is decreased in the development of GBM.

\section{miR-26b expression is inversely proportional to COX-2 expression}

To elucidate the biological relationship between miR-26b and COX-2 in cancer tissues of GBM, we analyzed the correlation between miR-26b and COX2 mRNA, and COX-2 protein. As shown in Figure 4, the linear regression analysis revealed that the miR-26b expression was negatively associated with the expression of COX-2 mRNA in 30 cases of GBM specimens $(p=0.001)$ (Figure 4a). As expected, miR-26b expression was also negatively associated with the expression of COX-2 protein in 30 cases of GBM specimens $(p=0.012)$ (Figure 4b).

\section{miR-26b inhibits proliferation and invasion of GBM cells}

To explore the role of miR-26b in the biological progression of GBM, we firstly explored the effect of miR-26b mimics on the overexpression of miR-26b in U87 and U251 cells, and confirmed that miR-26b mimics enhanced the expression of miR-26b (Figure 5a, 5b). Afterwards, we examined proliferation and invasion of GBM cells with different treatment. MTT assay revealed that U87 and U251 cells with miR-26b overexpression had

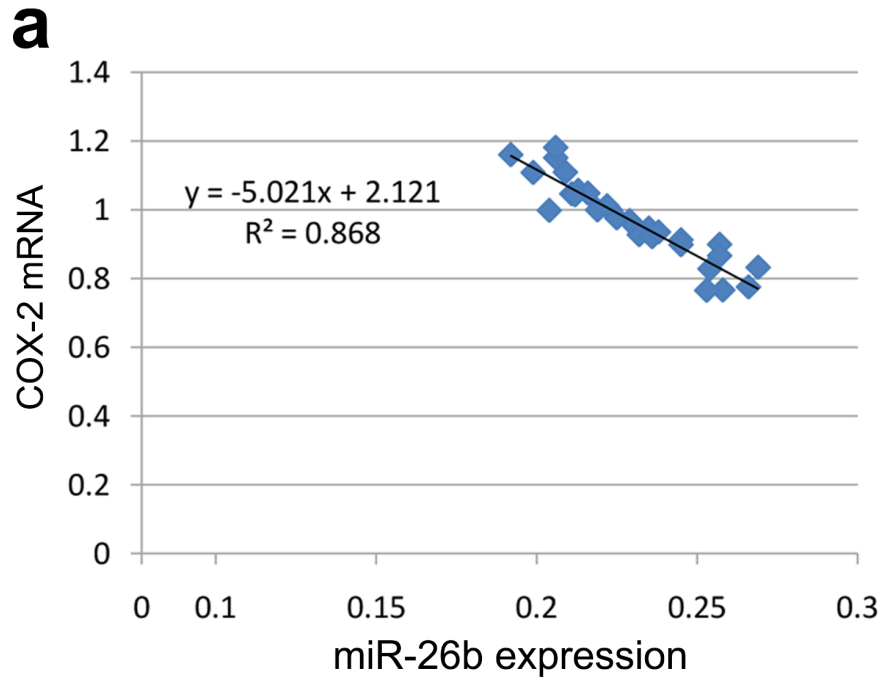

obviously lower growth rate, however the growth rate of control cells was not changed (Figure 5c, 5d). Besides, we also observed that the ectopic expression of miR-26b extremely decreased the invasion capacity of U87 and U251 cells (Figure 6a), which was indicated based on reduced colony numbers and sizes. These findings indicate that miR-26b overexpression inhibits cell proliferation and cell invasion in the development of GBM. As expected, flow cytometry analysis revealed that ectopic miR-26b expression obviously reduced the percentage of U87 and U251 cells in the S and G2/M stage, at the same time, ectopic miR-26b expression increased the percentage of U87 and U251 cells in the G1/G0 stage (Figure 6b), indicating that miR-26b overexpression indeed inhibits the proliferation of GBM cells by inducing cell cycle arrest. Notably, the expression of Bcl-2 and cyclin D1 was also significantly reduced but the expression of Bax was obviously increased in U87 and U251 cells transfected with miR-26b mimics (Figure 6c).

\section{COX-2 is a direct target of miR-26b}

According to recent studies, we used putative miRNA analysis software or website (TargetScan, Pictar, miRANDA) to demonstrate that COX-2 may be a potential target for miR-26b. In view of prediction results, our western blot analysis revealed that miR-26b mimics decreased the expression of COX-2 protein in U87 and U251 cells (Figure 6c), suggesting the inhibition effects of miR-26b on cell proliferation probably via targeting the expression of COX-2. Using COX-2 mutation, we found that ectopic miR-26b expression indeed decreased the expression of COX-2 in cells transfected with 3'UTR-WT of COX-2, however the expression of COX-2 in cells transfected with 3 'UTR-

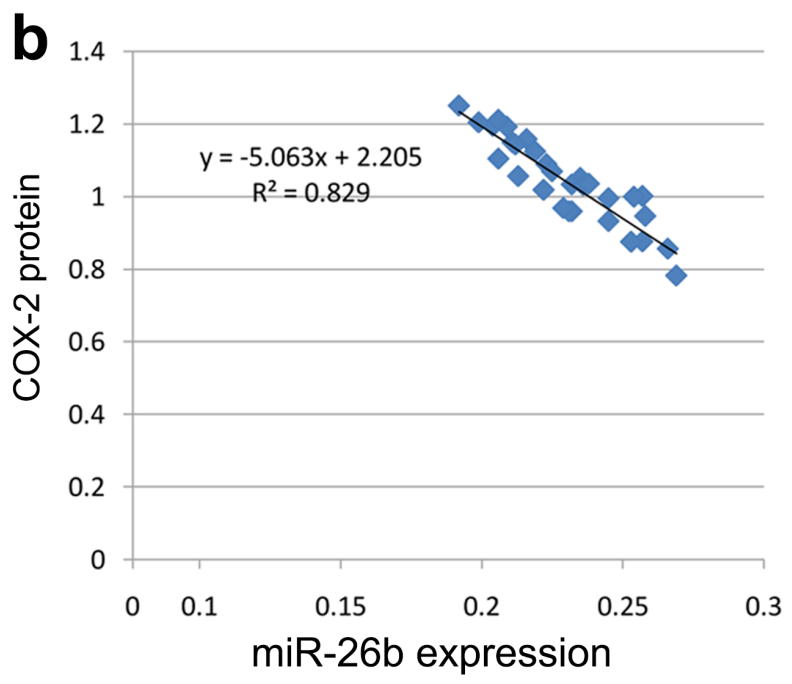

Figure 4: The correlation analysis between miR-26b and COX-2 in GBM tissues. a. The correlation between miR-26b and COX-2 mRNA expression in 30 GBM tissues was analyzed using linear regression. b. The correlation between miR-26b and COX-2 protein expression in $30 \mathrm{GBM}$ tissues was analyzed using linear regression with Excel statistics software. 

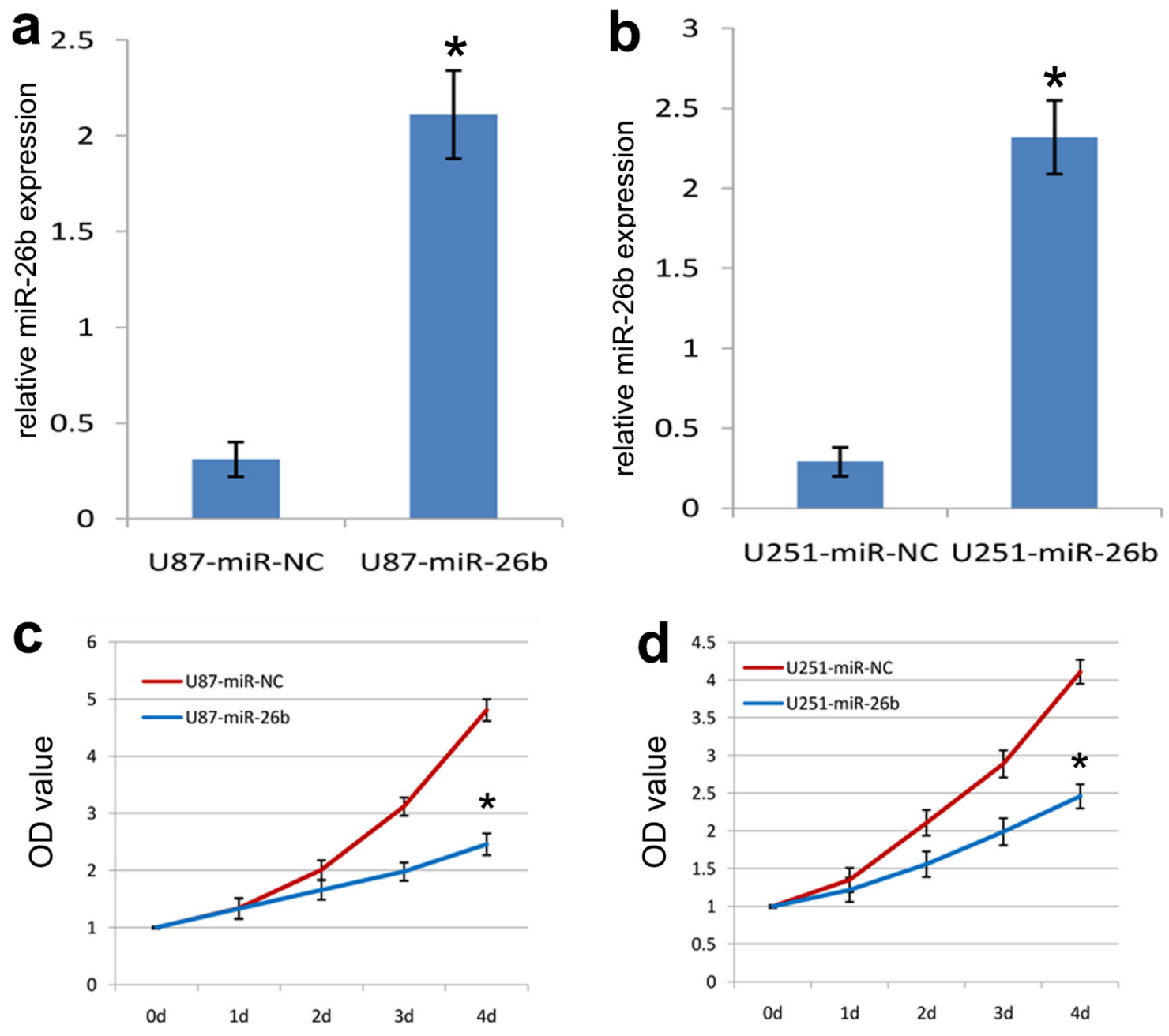

Figure 5: miR-26b inhibits GBM cell proliferation. a. Relative miR-26b expression in U87 cells was measured after the cells were transfected with miR-26b mimics or negative control (NC) miRNA using real-time RT-PCR. b. Relative miR-26b expression in U251 cells was measured after the cells were transfected with miR-26b mimics or NC miRNA using real-time RT-PCR. c. Cell proliferation was measured using a MMT assay. U87 cells were transfected with miR-26b mimics or NC control miRNA. d. MTT assays revealed that upregulation of miR-26b reduced cell growth compared to NC-transfected cells. Each bar represents the mean of three independent experiments. ${ }^{*} \mathrm{p}<0.001$, compared with control, one-way ANOVA.
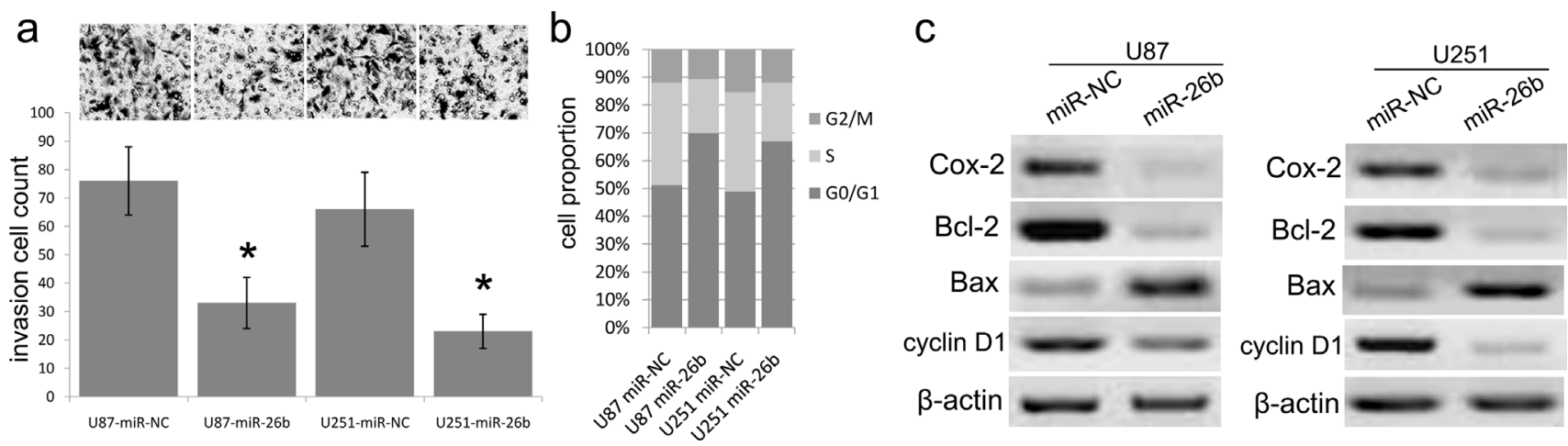

Figure 6: miR-26b inhibits GBM cell tumorigenicity and invasion. a. Upregulation of miR-26b reduced GBM cell tumorigenicity as determined by invasion assay. Representative micrographs and quantification of colonies that were larger than $0.1 \mathrm{~mm}$ were scored. Each bar represents the mean of three independent experiments. ${ }^{*} \mathrm{p}<0.001$, compared with control, one-way ANOVA. b. Flow cytometric analysis of indicated GBM cells after transfection with NC or miR-26b. The percentage of cell cycle was indicated. c. Western blotting analysis of expression of COX-2, Bcl-2, Bax and cyclin D1 in indicated cells. $\beta$-actin served as the loading control. 
MUT of COX-2 was not altered, which suggested that miR-26b affected the expression of COX-2 by binding the 3'UTR of COX-2 (Figure 7a, 7b). Besides, we also observed that overexpression of miR-26b could decrease the luciferase activity of COX-2-3'UTR-WT in a consistent and dose-dependent fashion, rather than the luciferase activity of COX-2-3'UTR-MUT (Figure $7 \mathrm{c}, 7 \mathrm{~d})$, which indicated that COX-2 was a direct target of miR-26b.

\section{DISCUSSION}

MicroRNAs have been reported as a kind of small RNA sequence that affect the transcription expression of some related mRNA or gene by binding specific sequences of mRNA. To date, miRNAs have been demonstrated as a useful regulator in the development of some tumors, including cell growth, apoptosis, differentiation, invasiveness and migration, and usually serve as a kind of tumor suppressors [7-11]. It has been reported that the COX-2 expression can be found to be increased in a wide variety of tumors, such as human colorectal cancer, liver cancer, cervical cancer, and pancreatic cancer [16-19]. Some studies indicated that the expression of COX-2 has been thought as a useful tumor biomarker, and COX-2 exhibited the increased invasion potential in cancers. In the present study, we investigated the expression model and molecular role of miR-26b and COX-2 in human GBM tissues and cells.

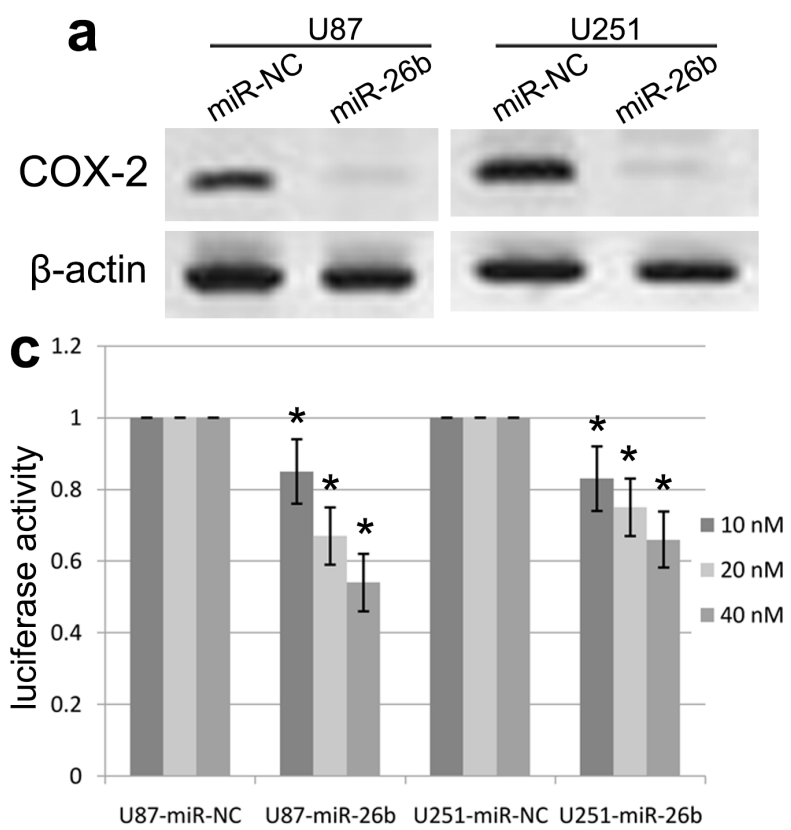

In this work, we demonstrate that the expression of COX-2 mRNA was obviously changed in human GBM cell lines and samples as compared to their adjacent nontumor tissues. The expression of COX-2 protein was also altered in human GBM cell lines and samples as compared with their adjacent non-tumor samples. Most importantly, the linear regression analysis revealed that the expression of miR-26b was negatively related with the expression of COX-2 mRNA or protein. Consistent with recently published articles, the expression of miR$26 \mathrm{~b}$ might be recommended as a tumor suppressor in breast cancer, which could act as a prognosis biomarker and a treatment target for breast cancer patients [20]. These findings suggested that miR-26b/COX-2 pathway is indeed involved in the development of GBM.

Furthermore, the overexpression of miR-26b reduced GBM cell proliferation and cell cycle progression, indicating that in-vivo overexpression of miR-26b may serve as a tumor suppressor, thus downregulation of miR-26b will facilitate the biological progression of GBM. Consistent with our study, it has been reported that miR-26b can also affect cell growth and invasiveness of colorectal cancer, but the inhibition effect of miR-26b can be partially attenuated by the enhancement of the target expression [21]. Some studies also found that miR-26b is able to inhibit the NF- $\kappa \mathrm{B}$ signaling, and affects the expression of TAK1 and TAB3, which further sensitizes the doxorubicin-induced liver cancer cell apoptosis [22]. Another report also indicated that the expression

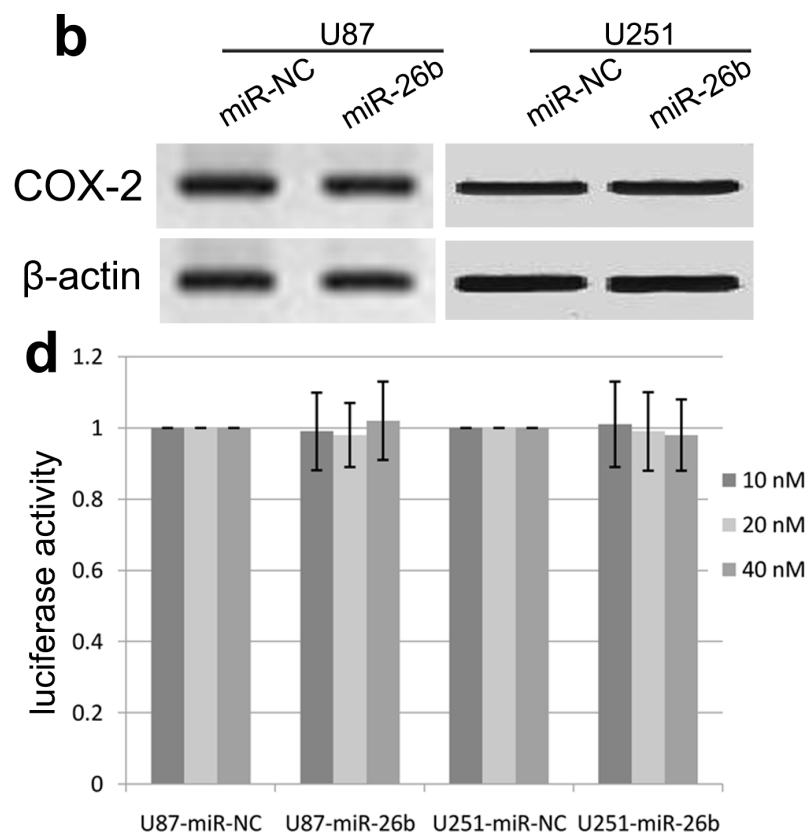

Figure 7: miR-26b downregulates COX-2 by directly targeting 3'-UTRs. Western blotting analysis of COX-2 expression was conducted in indicated cells transfected with the COX-2-3'UTR-wt reporter a. or the COX-2-3'UTR-mut reporter b. Luciferase assay was carried out in indicated cells transfected with the COX-2-3'UTR-wt reporter c. or the COX-2-3'UTR-mut d. reporter with increasing amounts $(10,20,40 \mathrm{nM})$ of miR-26b mimic, or NC miRNAs. Each bar represents the mean $\pm \mathrm{SEM}$ of three independent experiments. ${ }^{*} p<0.001$, compared with control, one-way ANOVA. 
of miR-26 can activate the pRb protein, and then block the G1/S-phase transition [23]. Overall, our study offered the latest evidence that the expression of miR-26b is significantly under expressed and exerts its anti-tumor role in the development of GBM by directly affecting the transcription of COX-2.

Notably, it has been reported that COX-2 inhibitors have the capacity of enhancing the sensitivity of tumors to radia-/chemotherapy based on some animal model experiments. For example, the recent study identified that administration with COX-2 inhibitor E-6087 could promote and facilitate apoptosis response of gliomas to the radiation intervention in mice models. At the same time, the radiation treatment combined with COX-2 inhibitor E-6087 not only promoted cell apoptosis, bur also affected the formation of murine microvascular systems [24, 25]. In addition, in vitro also identified that the antitumor effect of radiation treatment on murine intracranial glioma growth is enhanced by the inhibition of COX-2 [26, 27]. Thus, it is plausible that up-regulated miR-26b may sensitize GBM to chemotherapy-induced apoptosis by inhibiting the expression of COX-2.

In summary, in the present study, a new important role of miR-26b was identified, and miR-26b was demonstrated to regulate the tumorigenicity of GBM. The aberrant expression of miR-26b was associated with the proliferation and invasion of GBM. In addition to these findings, we also further ensured that miR$26 \mathrm{~b}$ binds the 3'-UTR of the COX-2 mRNA, and then reduced the expression level of COX-2. Our findings will provide a new insight for the treatment of GBM patients.

\section{MATERIALS AND METHODS}

\section{Ethical statement}

For the use of clinical materials for research purposes, samples were obtained with prior written informed consents from the patients and approval from the Institutional Research Ethical Committees of Yidu Central Hospital, Weifang Medical University.

\section{Cell culture and tissues}

Human GBM cell lines T98G, U87 and U251 were purchased from American Type Culture Collection (Manassas, VA). All cells were cultured in DMEM, 10\% fetal bovine serum (Gibco, Grand Island, NY, USA) and $1 \%$ penicillin-streptomycin (Gibco, Grand Island, NY, USA) at $37^{\circ} \mathrm{C}$ in a humidified atmosphere under $5 \% \mathrm{CO} 2$. In this study, 30 GBM samples were randomly chosen from the Department of Pathology in Shandong Cancer Hospital and Yidu Central Hospital of Weifang Medical University from January 2011 to June 2014. All samples were fixed with $10 \%$ formaldehyde and embedded with paraffin. Informed consent was obtained from all of the patients.

\section{COX-2 3'-UTR and miR-26b mimics construct}

COX-2 3'-UTR constructs were PCR amplified using cDNA encoding COX-2 as templates, and subcloned into pGL3-basic vector for luciferase reporter assay. PCR with Quickchange site directed mutagenesis kit from Qiagen (USA) were used for construction of mutant COX-2 3'-UTR. The miRNA mimics (ago-miR-26b), and negative controls of miR-26b, purchased from RiboBio (Ribo-Bio, Guangzhou, China) were transfected at final concentration of $40 \mathrm{nM}$. The FuGENE HD transfection agent (Promega, USA) was used according to the manufacturer's instructions.

\section{Quantitative real-time RT-PCR analysis}

Total RNA was isolated by using TRIzol (Invitrogen, USA). The concentration and quality of each RNA sample was determined using the A260/A280 spectrophotometric reading. The cDNA was synthesized with TaqMan reverse transcription reagents (Applied Biosystems, Branchbury, NJ, USA), following the manufacturer's recommendations. Real-time PCR was carried out using 7500 fast Real-time PCR System (Applied Biosystems). Reactions were run in three independent experiments. The relative fold changes in mRNA and miR-26b expression were calculated using the $2-\Delta \Delta C t$ method. Besides the relative expression was also normalized to GAPDH. The primer sequences were shown.

COX-2 primers:

Forward: 5'-CCTGGTCTGATGATGTATGC-3', Reverse: 5'-GTATGAGTCTGCTGGTTTGG-3'; GAPDH primers:

Forward: 5'-TTGGTATCGTGGAAGGACTCA-3'; Reverse: 5'-TGTCATCATATTTGGCAGGTT-3'. miR-26b primers:

Forward: 5'-CCGGAATTCCGGATGGGAATTGG ATACAT-3',

Reverse: 5'-ATTGCGGCCGCAGCTACCCTGAC CACTGCTGC-3';

U6 primers:

Forward 5'-CTCGCTTCGGCAGCACA-3', Reverse 5'-AACG CTTCACGAATTTGCGT-3';

RT-PCR for miR-26b was performed using real-time PCR Universal Reagent (GenePharma). U6 was used as an internal control.

\section{Western blot analysis}

Protein was extracted from cells using $1 \times$ radioimmunoprecipitation assay lysis buffer (Santa Cruz Biotechnology), subjected to SDS-PAGE, transferred to polyvinylidene difluoride membrane. Membranes were blocked for 2 hour in 5\% skimmed milk and probed with 
antibody against human COX-2, Bcl-2, Bax and cyclin D1 (Abcam, Cambridge, UK, 1:1000 dilution) or $\beta$-actin (Santa Cruz Biotech, Santa Cruz, CA, 1:1000 dilution) overnight at $4^{\circ} \mathrm{C}$, and then with peroxidase-conjugated secondary antibody (Santa Cruz Biotech, Santa Cruz, CA, 1:1000 dilution), visualized by chemiluminescence (GE, Fairfield, CT, USA).

\section{Luciferase assays}

Cells were seeded in triplicate in 24-well plates and allowed to settle for $24 \mathrm{~h} .100 \mathrm{ng}$ of pGL3-COX2-3'UTR (wt/mut) or control-luciferase plasmid plus 1 ng pRL-TK renilla plasmid (Promega, Madison, WI) were transfected into cells using Lipofectamine 2000 (Invitrogen Co., Carlsbad, CA) according to the manufacturer's recommendations. Luciferase and renilla signals were measured $48 \mathrm{~h}$ after transfection using the Dual Luciferase Reporter Assay Kit (Promega, Madison, WI) according to the manufacturer's protocol. Three independent experiments were performed and the data are presented as the mean \pm SEM.

\section{Flow cytometry analysis}

Cells were harvested by trypsinization, washed in ice-cold PBS, and fixed in $80 \%$ ice-cold ethanol in PBS. Before staining, cells were sedimented in a chilled centrifuge and resuspended in cold PBS. Bovine pancreatic RNase (Sigma-Aldrich) was added to a final concentration of $2 \mathrm{mg} / \mathrm{ml}$, and cells were incubated at $37^{\circ} \mathrm{C}$ for $30 \mathrm{~min}$, followed by incubation with $20 \mathrm{mg} / \mathrm{ml}$ of propidium iodide (Sigma-Aldrich) for $20 \mathrm{~min}$ at room temperature. Cell cycle profiles of cells were analyzed using a FAGBMC alibur flow cytometer (BD Biosciences).

\section{3-(4, 5-dimethyl-2-thiazolyl)-2, 5-diphenyl-2H- tetrazolium bromide (MTT)}

Cells, seeded on 96-well plates, were stained at indicated time points with $100 \mathrm{ml}$ sterile MTT dye $(0.5$ $\mathrm{mg} / \mathrm{ml}$, Sigma) for $4 \mathrm{~h}$ at $37^{\circ} \mathrm{C}$, followed by removal of the culture medium and addition of $150 \mathrm{ml}$ of dimethyl sulphoxide (DMSO) (Sigma, St. Louis, MO, USA). The absorbance was measured at $570 \mathrm{~nm}$, with $655 \mathrm{~nm}$ as the reference wave length. All experiments were performed in triplicate.

\section{Invasion assay}

For invasion assay, cells were added to the upper well chamber and coated with Matrigel (BD Biosciences) for 24 hours. Then, cells on the upper membrane surface were removed by careful wiping with a cotton swab, and the filters were fixed with $95 \%$ ethanol and stained with $0.2 \%$ crystal violet solution (Sigma) and then counted.

\section{Statistical analysis}

Statistical analyses were carried using the SPSS 17.0 program. An independent samples test was run to analyze the significance of the differences found. P-values less than 0.05 were considered to be statistically significant. All data are presented as the mean \pm SEM.

\section{ACKNOWLEDGMENTS}

We greatly thank other members in our lab for valuable suggestions and writing.

\section{CONFLICTS OF INTEREST}

The authors declare no conflicts of interest.

\section{REFERENCES}

1. Yang X, Lv S, Liu Y, et al. The Clinical Utility of Matrix Metalloproteinase 9 in Evaluating Pathological Grade and Prognosis of Glioma Patients: A Meta-Analysis. Mol Neurobiol. 2015; 52:38-44.

2. Yang X, Li D, Cheng S, et al. The correlation of bone morphogenetic protein 2 with poor prognosis in glioma patients. Tumour Biol. 2014; 35:11091-5.

3. Dai C, Lv S, Shi R, et al. Nuclear Protein C23 on the Cell Surface Plays an Important Role in Activation of CXCR4 Signaling in Glioblastoma. Mol Neurobiol. 2015; 52:1521-6.

4. Franceschi S, Mazzanti CM, Lessi F, et al. Investigating molecular alterations to profile short- and long-term recurrence-free survival in patients with primary glioblastoma. Oncol Lett 2015; 10:3599-3606.

5. He Y, Zhao C, Liu Y, et al. MiR-124 Functions as a Tumor Suppressor via Targeting hCLOCK1 in Glioblastoma. Mol Neurobiol. 2016. [Epub ahead of print]

6. Yao C, Li P, Song H, et al. CXCL12/CXCR4 Axis Upregulates Twist to Induce EMT in Human Glioblastoma. Mol Neurobiol. 2015. [Epub ahead of print]

7. Muluhngwi $\mathrm{P}$, Klinge CM. Roles for miRNAs in endocrine resistance in breast cancer. Endocr Relat Cancer. 2015; 22:R279-300.

8. Kagiya T. MicroRNAs and Osteolytic Bone Metastasis: The Roles of MicroRNAs in Tumor-Induced Osteoclast Differentiation. J Clin Med. 2015; 4:1741-52.

9. Liu H, Ren G, Zhu L, et al. The upregulation of miRNA146a inhibited biological behaviors of ESCC through inhibition of IRS2. Tumour Biol. 2015. [Epub ahead of print] PMID: 26508028.

10. Luna-Aguirre CM, de la Luz Martinez-Fierro M, MarAguilar F, et al. Circulating microRNA expression profile in B-cell acute lymphoblastic leukemia. Cancer Biomark. 2015; 15:299-310. 
11. Sampson VB, Yoo S, Kumar A, Vetter NS, et al. MicroRNAs and Potential Targets in Osteosarcoma: Review. Front Pediatr. 2015; 3:69.

12. Lv S, Sun B, Dai C, et al. The Downregulation of MicroRNA-146a Modulates TGF- $\beta$ Signaling Pathways Activity in Glioblastoma. Mol Neurobiol. 2015; 52:1257-62.

13. Verghese ET, Drury R, Green CA, et al. MiR-26b is down-regulated in carcinoma-associated fibroblasts from ER-positive breast cancers leading to enhanced cell migration and invasion. J Pathol. 2013; 231:388-99

14. Li J, Kong X, Zhang J, et al. MiRNA-26b inhibits proliferation by targeting PTGS2 in breast cancer. Cancer Cell Int. 2013; 13:7.

15. Zhu Y, Lu Y, Zhang Q, et al. MicroRNA-26a/b and their host genes cooperate to inhibit the G1/S transition by activating the $\mathrm{pRb}$ protein. Nucleic Acids Res. 2012; 40:4615-25.

16. Bayomi EA, Barakat AB, El-Bassuoni MA, et al. Cyclooxygenase-2 expression is associated with elevated aspartate aminotransferase level in hepatocellular carcinoma. J Cancer Res Ther. 2015; 11:786-92.

17. Che XH, Chen CL, Ye XL, et al. Dual inhibition of COX2/5-LOX blocks colon cancer proliferation, migration and invasion in vitro. Oncol Rep. 2016; 35:1680-8.

18. Su HJ, Zhang Y, Zhang L, et al. Methylation status of COX-2 in blood leukocyte DNA and risk of gastric cancer in a high-risk Chinese population. BMC Cancer. 2015; 15:979.

19. Yokouchi H, Kanazawa K. Revisiting the role of COX-2 inhibitor for non-small cell lung cancer. Transl Lung Cancer Res. 2015; 4:660-4.
20. Li J, Li X, Kong X, et al. MiRNA-26b inhibits cellular proliferation by targeting CDK8 in breast cancer. Int J Clin Exp Med. 2014; 7:558-65.

21. Zhang C, Tong J, Huang G. Nicotinamide phosphoribosyl transferase (Nampt) is a target of microRNA-26b in colorectal cancer cells. PLoS One. 2013; 8:e69963.

22. Zhao N, Wang R, Zhou L, et al. MicroRNA-26b suppresses the NF-kappaB signaling and enhances the chemosensitivity of hepatocellular carcinoma cells by targeting TAK1 and TAB3. Mol Cancer. 2014; 13:35.

23. Ying Zhu, Yang Lu, Qi Zhang, et al. MicroRNA-26a/b and their host genes cooperate to inhibit the G1/S transition by activating the pRb protein. Nucleic Acids Res. 2012; 40:4615-4625.

24. Wagemakers M, van der Wal GE, Cuberes R, et al. COX-2 Inhibition Combined with Radiation Reduces Orthotopic Glioma Outgrowth by Targeting the Tumor Vasculature. Transl Oncol. 2009; 2:1-7.

25. Petersen C, Petersen S, Milas L, et al. Enhancement of intrinsic tumor cell radiosensitivity induced by a selective cyclooxygenase-2 inhibitor. Clin Cancer Res. 2000; 6:2513-20.

26. Pyo H, Choy H, Amorino GP, et al. A selective cyclooxygenase-2 inhibitor, NS-398, enhances the effect of radiation in vitro and in vivo preferentially on the cells that express cyclooxygenase-2. Clin Cancer Res. 2001; 7:2998-3005.

27. Shin YK, Park JS, Kim HS, et al. Radiosensitivity enhancement by celecoxib, a cyclooxygenase (COX)-2 selective inhibitor, via COX-2-dependent cell cycle regulation on human cancer cells expressing differential COX-2 levels. Cancer Res. 2005; 65:9501-9. 